\title{
3-D In Vitro Acoustic Super-Resolution and Super-Resolved Velocity Mapping Using Microbubbles
}

\author{
Kirsten Christensen-Jeffries, Member, IEEE, Jemma Brown, Student Member, IEEE, Paul Aljabar, \\ Mengxing Tang, Member, IEEE, Christopher Dunsby, Robert J. Eckersley, Senior Member, IEEE.
}

\begin{abstract}
Standard clinical ultrasound (US) imaging frequencies are unable to resolve microvascular structures due to the fundamental diffraction limit of US waves. Recent demonstrations of 2D super-resolution both in vitro and in vivo have demonstrated that fine vascular structures can be visualized using acoustic single bubble localization. Visualization of more complex and disordered 3D vasculature, such as that of a tumor, requires an acquisition strategy which can additionally localize bubbles in the elevational plane with high precision in order to generate super-resolution in all three dimensions. Furthermore, a particular challenge lies in the need to provide this level of visualization with minimal acquisition time. In this work, we develop a fast, coherent US imaging tool for microbubble localization in 3D using a pair of US transducers positioned at $90^{\circ}$. This allowed detection of point scatterer signals in 3 dimensions with average precisions equal to $1.9 \mu \mathrm{m}$ in axial and elevational planes, and $11 \mu \mathrm{m}$ in the lateral plane, compared to the diffraction limited point spread function full widths at half maximum of $488 \mu \mathrm{m}, 1188 \mu \mathrm{m}$ and $953 \mu \mathrm{m}$ of the original imaging system with a single transducer. Visualization and velocity mapping of $3 \mathrm{D}$ in vitro structures was demonstrated far beyond the diffraction limit. The capability to measure the complete flow pattern of blood vessels associated with disease at depth would ultimately enable analysis of in vivo microvascular morphology, blood flow dynamics and occlusions resulting from disease states.
\end{abstract}

This research was supported by the Department of Health via the National Institute for Health Research (NIHR) comprehensive Biomedical Research Center award to Guy's and St Thomas' NHS Foundation Trust in partnership with King's College London and King's College Hospital NHS Foundation Trust. The Centre of Excellence in Medical Engineering funded by the Wellcome Trust and EPSRC under grant number WT 088641/Z/09/Z, EPSRC grant number EP/N14855/1, and EPSRC grant number EP/M011933/1. The views expressed are those of the author(s) and not necessarily those of the NHS, the NIHR or the Department of Health.

K. Christensen-Jeffries, J. Brown, P. Aljabar, and R. J. Eckersley are with the Biomedical Engineering Department, Division of Imaging Sciences, Kings College London, London, UK (e-mail: kirsten.christensen-jeffries@kcl.ac.uk, jemma.brown@kcl.ac.uk, paul.aljabar@kcl.ac.uk and robert.eckersley@kcl.ac.uk, phone: 02071888371).

M. X. Tang is with the Department of Bioengineering, Imperial College London, London, SW7 2AZ, UK (email: mengxing.tang@imperial.ac.uk).

C. Dunsby is with the Department of Physics Imperial College London, London, SW7 2AZ, UK and the Centre for Pathology, Imperial College London, London, W12 0NN, UK (email: christopher.dunsby@imperial.ac.uk).
Index Terms - Microbubbles, microvasculature, resolution, ultrasound.

\section{INTRODUCTION}

$\mathrm{T}$ HE structure and flow of the microcirculation reflects the requirements and conditions of local cells and tissue. This assertion underscores the importance of visualizing the presence, structure and function of blood vessels. Architectural changes in the micro-vascular structure, as well as variations in vascular flow can be a marker of pathological, damaged or dysfunctional tissue, such as ischemia [1], peripheral arterial disease (PAD) [2]-[5], and coronary heart disease [6], while an increase can indicate rapid and uncontrollable cell growth or proliferation caused by cancer and metastasis [7]-[10]. Detecting such microscopic changes in vivo using non-invasive high-resolution imaging would allow early clinical intervention and would provide a means of closely monitoring the treatment of such diseases. However, small vessel sizes and low blood flows make assessing the microcirculation challenging [11].

Through the development of microbubbles as US contrast agents [12], [13] and the advent of advanced techniques to exploit their nonlinear response, the specificity and sensitivity of clinical US vascular imaging has improved [14]. However, the limitation on conventional US resolution by diffraction means imaging at the micrometer scale is still required. High frequency US has been developed as a means of obtaining high-resolution imaging [11], however these techniques have limited application due to the inherent compromise between higher frequencies and limited penetration depth. Recently developed ultrasound super-resolution (US-SR) imaging has demonstrated spatially resolved maps of fine microvessels in vitro [15] and in vivo [16] by imaging the signals from spatially isolated microbubbles flowing within the circulation. This has been achieved using an unmodified clinical US system operating in a standard contrast enhanced mode that separates microbubbles from background by detecting the non-linear acoustic response of the microbubbles. This work has demonstrated significant improvement in spatial resolution even with the restricted data accessibility provided by these 
clinical systems.

The recent introduction of ultrasonic plane-wave imaging has enabled fast frame rates often over 1000 frames per second [17]-[20]. Super-resolution US imaging has also been demonstrated by using these high frame-rates to identify bubbles from background in RF data based on their motion and/or disruption over time [21], [22]. A bubble-motion based super-resolution imaging approach has also been demonstrated using a clinical US scanner [23]. A third approach for identifying single bubbles in ex vivo samples is to acquire an US image of the sample with no microbubbles present and to then subtract this static background from frames acquired with microbubbles present [24].

Much of this previous work has been based around the use of 1-D linear array transducers that has restricted the superresolved image information to $2 \mathrm{D}$ [15], [16], [23]. Here, as well as in the case of 3-D volume acquisition using mechanical scanning in the third dimension [24], the elevational resolution remains at the conventional diffraction limit. O'Reilly et al. [24] used a hemi-spherical sparse array to achieve true 3-D super-resolution imaging through an ex vivo skull phantom, but the image acquisition rate was relatively slow (PRF $10 \mathrm{~Hz}$ ), and the transmit focus was steered through the imaging volume with a step size of $2 \mathrm{~mm}$. In work by Desailly et al. [21], a 2-D matrix array was applied to demonstrate super-resolution imaging in $3-\mathrm{D}$, but this approach used a bespoke matrix array transducer and details of the data acquisition are unclear. Furthermore, the resulting elevational resolution achieved is not presented.

A particular challenge lies in the need to provide volumetric super-resolution visualization with minimal acquisition time and maximum spatial resolution. In this paper, we demonstrate a compounding strategy using a synchronized pair of orthogonal linear US arrays for 3D bubble localization. The diffraction limited resolution and the 3D localization precision of this US imaging system are measured, before 3-D localization experimental results are obtained using in vitro phantoms. Three dimensional acoustic super-resolution is then demonstrated using $3 \mathrm{D}$ in vitro flow phantoms.

\section{THEORY}

\section{A. Principles of the Method}

Acoustic scattering from diagnostic microbubbles is dominated by the active emission of sound due to the change in volume of the scatterers [25] and can therefore be assumed to be spherically symmetric. An imaging configuration has thus been designed to detect scattering effects in two complementary imaging planes. The proposed imaging strategy involves the transmission and reception of plane wave contrast pulse sequences [26] from a programmable 1D linear array transducer, termed the 'active' system, with simultaneous detection of the scattered signals from gas-filled microbubbles by a second temporally synchronized, system, termed the 'passive' system. Passive cavitation detectors used for passive acoustic mapping implement the same principles to that used in this study [27].

In this method, a pulse of US is emitted from the active transducer and insonates a low concentration of microbubbles. If the path of the emitted pulse insonifies a bubble which lies within the imaging plane of the passive imaging transducer, then sufficient information can be obtained to localize the bubble in 3D space. The region defined by the overlapping imaging planes therefore determines the field of view in which 3D localization is possible, as illustrated in Fig. 1.

Provided that temporal synchronization of the two imaging systems is achieved, the time of arrival of echoes from moving objects recorded at both transducers can be used to locate a scatterer. The time taken for the scattered signals to arrive at the passive imaging transducer depends on the length of the acoustic path taken. This path length can be estimated by assuming a constant speed of sound in the medium of interest and calculating the time interval between scattering of sound by the bubble and detection of signal by the passive scanner. The time taken to reach the bubble can be calculated from the signal detected by the active scanner.

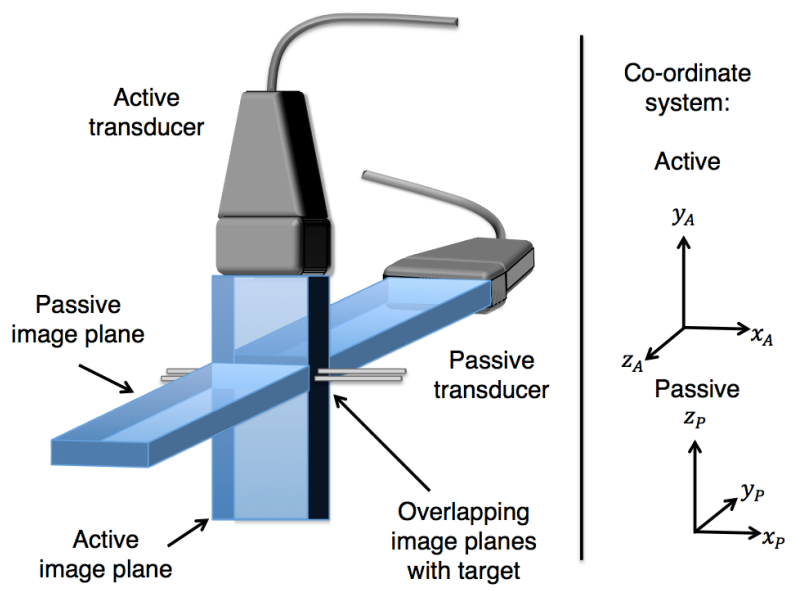

Fig. 1. Illustration of active and passive transducer configuration when orthogonally positioned. The region defined by the overlapping imaging planes therefore determines the field of view in which $3 \mathrm{D}$ localization of microvessel tubes is possible. Independent co-ordinate systems for the active and passive transducers are displayed on the right hand side.

As such, a point-like scatterer positioned at $r_{s}=\left(x_{s}, y_{s}, z_{s}\right)$ in the active coordinate system will scatter US energy radially assuming a monopole scattering behavior. The 1-D active transducer array $T_{A}$ will provide visualisation of the scatterer in a 2-D image plane and enable measurement of its coordinates in the directions $\left(x_{A}, y_{A}\right)$ (see co-ordinate system in Fig. 1). In this conventional imaging system, coordinate $z_{S}$ can be localized only to within the elevational resolution of the system. Positioning transducer $T_{P}$ orthogonally, or at an angle, to $T_{A}$ allows in plane measurement in the directions of $\left(x_{P}, y_{P}\right)$. Coordinate $z_{S}$ can therefore be determined, in the orthogonal case, to within the axial localization precision of the passive transducer from the measurement of $y_{P}$. Thus, the 
passive transducer enables higher precision localization within the elevational imaging plane of transducer $T_{A}$.

Conventional US imaging with line-by-line focused pulses limits the highest achievable frame rate. The use of plane wave transmissions enables faster frame rates and is able to insonify a large field of view with each transmission [18] [20]. Plane wave transmission was therefore implemented and adapted for nonlinear imaging to recover microbubble specific images.

\section{MATERIALS AND METHODS}

\section{A. Ultrasound Equipment and Acquisition}

Data were acquired using two Ultrasound Advanced Open Platform (ULA-OP) systems (MSD Lab, University of Florence, Italy). These are open platform, programmable US imaging systems with 64 independent arbitrary waveform generators able to simultaneously control up to 64 elements of a number of different element array probes with user-defined pulse sequences [28]. Each ULA-OP system was used to drive a LA332 imaging transducer (Esaote, Firenze, Italy). This 144 element linear array probe has a $6 \mathrm{~dB}$ bandwidth ranging from $2 \mathrm{MHz}$ to $7.5 \mathrm{MHz}$ bandwidth and a $0.254 \mathrm{~mm}$ pitch.

\section{B. Phantom Construction}

Phantoms developed for 3D US imaging were constructed using medium density paraffin gel wax [29]. These phantoms allow $90^{\circ}$ transducer positioning without the need to submerge the imaging probes deep into water. To create the phantom, firstly, paraffin gel wax was placed into a silicone mold using sterile gloves in a clean environment to limit the possibility of small fragments and dust particles embedding in the phantom. The speed of sound within the phantom was estimated to be $1425 \pm 3 \mathrm{~m} / \mathrm{s}$ using a reflection substitution technique.

In order to create a sub-diffraction sized linear scatterer within the phantom, a small air bubble was injected into the paraffin gel wax approximately 10 minutes after removing the phantom from the oven using a micro-pipette. Optical images were then acquired using a microscope where this air bubble was estimated to have a diameter of $133 \mu \mathrm{m}$. A second phantom was created in which a coiled cellulose tube with inner diameter of $200 \mu \mathrm{m}$ was embedded into the paraffin after the same amount of cooling time. This was then trimmed and inserted into $25 \mathrm{G}$ butterfly winged infusion needles at either end. An epoxy resin was used to secure the connections between the tubes and the needles.

\section{Ultrasound System Characterization - Estimation of Diffraction Limited Resolution}

The diffraction limited resolution of the imaging system under ideal conditions was investigated using $3 \mathrm{MHz}$ transmit frequencies with a single ULA-OP system. Focused B-Mode pulses were transmitted using a 3 cycle pulse with the system maximum amplitude, PRF of $4000 \mathrm{~Hz}$, and a focus distance of $18 \mathrm{~mm}$. The PSF of the point scatterer phantom was measured at depths between $13 \mathrm{~mm}$ to $23 \mathrm{~mm}$ in the axial direction, and across $12 \mathrm{~mm}$ laterally. Focused US data was acquired in IQ format. With the scatterer at the focus, 100 plane wave pulses were additionally transmitted and saved in pre-beamformed RF format in order to compare the PSF achieved using focused imaging, and delay and sum beamformed plane wave reconstruction. The resolution of the US system was measured as the FWHM of the beamformed signal in the lateral and axial directions.

The elevational resolution of the system was estimated by translating a wire target in $0.250 \mathrm{~mm}$ steps across the field of view at a depth of $18 \mathrm{~mm}$ using a micrometer stage and measuring the signal amplitude in the acquired IQ data. To account for the finite size of the wire in the measurement of the PSF, the FWHM value was deconvolved by the scatterer diameter to obtain new values of the PSF FWHM as performed in our previous work [16].

\section{Ultrasound System Characterization - Estimation of Localization Precision in 3-D}

To estimate the localization precision achievable using super-resolution, the orthogonal setup illustrated in Fig. 1 was constructed using two identical LA332 transducers each connected to individual ULA-OP systems. The systems were synchronized via the use of a single system clock source.

The point scatterer phantom was positioned within the overlapping imaging region of the orthogonal transducers at the focus. Three cycle, single angle plane waves were transmitted from the central 64 elements of the active transducer at $3 \mathrm{MHz}$ with a PRF equal to $4000 \mathrm{~Hz}$ and amplitude equal to 1 (a.u.). Coincident echo detections at the passive and active imaging probes were then used for $3 \mathrm{D}$ localization. The standard deviation of the localisation position over 100 frames provided an estimate of the localisation precision.

\section{E. In Vitro Microbubble Imaging}

Two $200 \mu \mathrm{m}$ internal diameter cellulose tubes (Hemophan $\AA$, Membrana) were held alongside each other within a distance smaller than the elevational resolution of the active transducer, estimated to be $1 \mathrm{~mm}$ (see Results). The apparatus was placed in a gas-equilibrated water bath at a depth of approximately $14 \mathrm{~mm}$. The ULA-OP systems were synchronized in the aforementioned orthogonal set-up with the tubes positioned within the volume $V$ defined by the overlapping imaging planes, estimated to be equal to

$$
\begin{gathered}
V=F O V_{x} \times F O V_{Z A} \times F O V_{Z P}, \\
V=15.4 \mathrm{~mm} \times 1.188 \mathrm{~mm} \times 1.188 \mathrm{~mm}, \\
V=21.7 \mathrm{~mm}^{3} .
\end{gathered}
$$

where $F O V_{x}$ is the lateral field of view, and $F O V_{Z A}$ and $\times F O V_{Z P}$ are the elevational field of view for the active and 
passive transducers respectively. A low concentration dilution of $100 \mu \mathrm{l} \mathrm{SonoVue}{ }^{\mathrm{TM}}$ in $600 \mathrm{ml}$ water was prepared which provided a suitable concentration for imaging spatially isolated microbubbles. The solution was drawn through both tubes at $50 \mu \mathrm{l} / \mathrm{min}$ with opposing flow directions.

The active ULA-OP system was driven at a frame rate of $400 \mathrm{~Hz}$ using amplitude modulation (AM) plane wave transmission using three different relative amplitudes of $1,-1$ and 0.5 (a.u.) at a transmit frequency of $3 \mathrm{MHz}$. Peak negative pressures at the target depth were estimated using hydrophone measurements to be 180 for 0.5 amplitude pulse, and $377 \mathrm{kPa}$ for full amplitude pulse. All 64 active elements were used in parallel on both ULA-OP systems. RF data was saved from both systems simultaneously. A single dataset consisted of a 15 second acquisition corresponding to 6000 individual frames, equivalent to $2000 \mathrm{AM}$ frames once combined. Five datasets were collected and processed for the final visualization.

\section{F. Post-Processing Procedures}

In previous work, localization of individual bubble signals involved calculating the centre of mass of an image formed by the microbubble to estimate its location [15], [16]. As discussed previously, when a small scatterer is insonified by a plane wave, it can be assumed to emit a spherical wave. In this work, scatterer localizations were estimated using two methods for comparison. In the first method, the peak of each RF data line from each element was found. Since a spherical wave emitted by a point scatterer can be approximated as a hyperbola when detected across parallel transducer array elements, the peaks detected in each channel line were used to fit a hyperbola across the lateral image plane. The position of the minimum in time of this hyperbola was used as the estimate of the location of the insonated point scatterer. In the second method, the received signal was beamformed offline to reconstruct an image of the point scatterer. The location of the scatterer was then estimated by calculating the centre of mass of the signal as performed in previous work [15], [16].

The localization precision for each method was measured to be the standard deviation of the localization positions over 100 frames. Average estimated localization precisions were implemented in rendering procedures for subsequent in vitro experiments. The SNR was calculated as the ratio of the average absolute intensity of the detected signal within the field of view, to the average absolute background signal.

For in vitro microbubble imaging, identical post-processing was performed for both active and passive data. To remove unwanted background signals, frames with a low total intensity considered not to contain a bubble were added to a rolling average of the previous 10 background frames that was then subtracted from the next frames containing a bubble. Next, an asymmetric median filter of size [2 $\times 8$ ] (time $\times$ space) was applied; this size was chosen to smooth noise variations across the RF signal frame without significantly blurring the data, which possessed higher resolution in the temporal direction.

A template-matching algorithm was then used to locate hyperbola structures in the data corresponding to individual bubbles by computing the normalized cross-correlation between the RF data and a reference signal or 'template'. The template was constructed by extracting the scatter signal from the previously acquired linear (non-contrast enhanced) point scatterer echo obtained from the air bubble inclusion in paraffin wax. The position of the maximum cross-correlation identified the approximate position of the scatterer in the data. So as not to bias the bubble localization by characteristics of the linear scatterer signal, a region of interest surrounding the maximum position of cross-correlation was extracted using a binary mask. The binary mask consisted of a region of pixels with a value of one which extended $4 \mu$ s above and below the fitted template position across the whole lateral width of the image, while the remaining pixels had a value of zero. This allowed extraction of the full axial extent of the detected bubble signals to incorporate phase information in the localization, while still isolating potential bubble signals from erroneous or noise related signals present in the rest of the data which may decrease the accuracy of the technique. The peaks of each RF data line within the masked frame were then fitted with a hyperbola; the point on the curve representing the minimum arrival time was estimated to represent the bubble position.

Coincident detections of plane wave echoes by both transducers were extracted. Active localizations provided measurement of the axial and lateral bubble positioning in $\left(x_{A}, y_{A}\right)$ while the passive system enabled localization within the active transducer's elevational plane, $\left(z_{A}\right)$. For each pair, a velocity vector was then calculated analogous to that performed in our previous work in 2-D [16], between $x_{l}{ }^{n}, y_{l}{ }^{n}$, $z_{l}^{n}$, and $x_{l}^{n-1}, y_{l}{ }^{n-1}, z_{l}^{n-l}$ and assigned to the centroid location $x_{l}^{n}, y_{l}^{n}, z_{l}^{n}$, in frame $n$.

\section{G. Image Generation}

A 3D rendering of the combined localizations was constructed by plotting each estimated bubble location, $r_{l}=$ $x_{l}, y_{l}, z_{l}$, as an ellipsoid with semi-axis lengths equal to the previously estimated localization precisions in the axial, lateral and elevational directions, $\sigma=\left(\sigma_{x_{l}}, \sigma_{y_{l}}, \sigma_{z_{l}}\right)$, given by

$$
\frac{\left(x-x_{l}\right)^{2}}{\sigma_{x}^{2}}+\frac{\left(y-y_{l}\right)^{2}}{\sigma_{y}^{2}}+\frac{\left(z-z_{l}\right)^{2}}{\sigma_{z}^{2}}=1,
$$

where $x, y$, and $z$ are the coordinates of any points on the surface of the ellipsoid. Microbubble tracking algorithms developed in our previous work [16] were extended for 3D implementation to determine the flow velocity within the microvessel structure. Intensity cross-correlations between each bubble signal in frame $n$ and each of the bubble signals found in frame $n-1$ were then calculated and the maximum cross correlation was found for each signal in frame $n$. A pair 
of signals were considered to come from the same bubble if the maximum cross-correlation exceeded an empirically determined threshold of 0.4 (significantly lower than that in previous work [16] due to the nature of the RF signals compared to beamformed, positive, envelope detected image data).

\section{RESUlts}

\section{A. Ultrasound System Characterization}

An example data frame from a single transducer operating in conventional B-Mode in which a point scatterer was positioned at a depth of $18 \mathrm{~mm}$ using $3 \mathrm{MHz}$ insonation frequency is shown in Fig. 2. Average measured FWHMs over a region in the field of view approximately $10 \mathrm{~mm} \times 12 \mathrm{~mm}$ in size using focused imaging (or over repeated measurements in the case of the elevational resolution) at a transmit frequency of $3 \mathrm{MHz}$ equate to $534 \pm 29 \mu \mathrm{m}, 953 \pm 103 \mu \mathrm{m}$ and $1188 \pm 160 \mu \mathrm{m}$ in the axial, lateral and elevational planes respectively. An improved axial resolution of $488 \mu \mathrm{m}$ was observed using plane wave beamforming. These measured values provide an approximate comparison for estimating the resolution improvement achieved after localizing image data. The wavelength of the transmission pulse, here equal to 500 $\mu \mathrm{m}$, also provides an indication of the system resolution which can be used to compare to the achieved super-resolution.

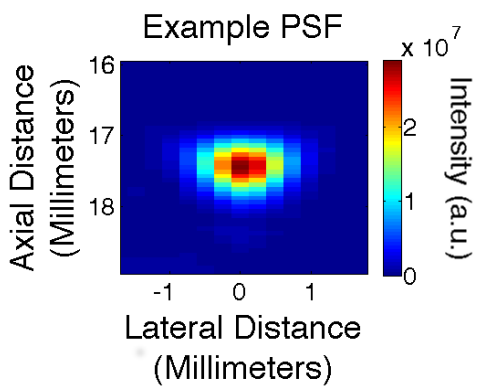

Fig. 2. An IQ image data frame at the transducer focus of $18 \mathrm{~mm}$ at $3 \mathrm{MHz}$ with the LA332 probe representing the diffraction limited in plane resolution of the imaging system. Average resolution estimations over the field of view are $534 \pm 29 \mu \mathrm{m}$ and $953 \pm 103 \mu \mathrm{m}$ respectively.

Localization precisions calculated across the entire field of view are displayed in Fig. 3. The average lateral localization precisions have an average of $11.0 \pm 25.0 \mu \mathrm{m}$, with a minimum of $6.9 \mu \mathrm{m}$ and maximum of $101.7 \mu \mathrm{m}$, as shown in Fig. 3a. The average axial localization precision is $1.9 \pm 1.6$ $\mu \mathrm{m}$, ranging from $0.7 \mu \mathrm{m}$ to $9.4 \mu \mathrm{m}$ at the edge of the field of view, see Fig. 3b. Signal-to-noise (SNR) values are shown in Fig. 3c.

Five hundred echoes were simultaneously detected from the single air bubble inclusion in paraffin gel by both the active and passive transducers. Fig. $4 a$ and $b$ display the coincident localizations within the co-ordinate system of each transducer. The axial component of the passive localizations enable the position of the scatterer to be determined within the active transducer's $\left(x_{A}, z_{A}\right)$ plane; the resulting 3D localization precision is thus shown in Fig. 4c. The diffraction-limited spatial resolution of a single transducer alone at $3 \mathrm{MHz}$ transmit frequency, measured from the PSF profiles at the focus in the axial and lateral directions, and the amplitude profile in the elevational direction are shown in Table 1 together with the estimated super-resolution localization precisions in the same three directions.

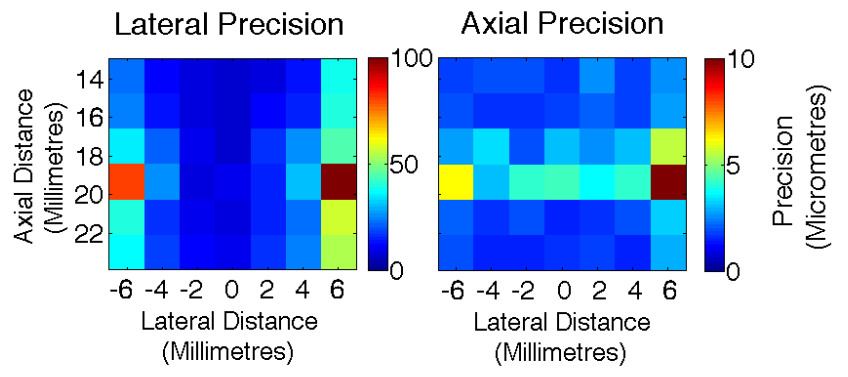

(a)

(b)

SNR

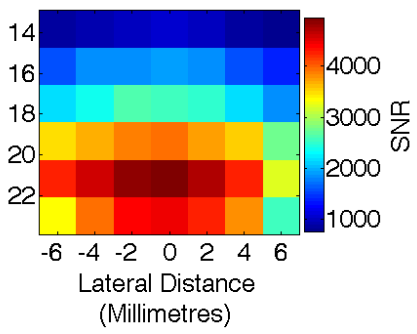

(c)

Fig. 3. Localization precision estimations using RF plane wave receive signals across the field of view at a transmit frequency of $3 \mathrm{MHz}$. (A) and (B) show the axial and lateral precisions respectively, and $(\mathrm{C})$ is the estimated signal-tonoise (SNR) of the receive signals.

\section{B. In Vitro Microbubble Imaging}

Fig. 5a displays the combined localizations from all acquired frames from contrast enhanced imaging of microbubble flowing through the phantom consisting of two adjacent tubes with opposing flow directions. The elevational component (along $Z_{A}$ ) of the measured bubble velocity remained relatively constant throughout the data so therefore the color of each ellipsoid displayed in the figure represents the lateral directional component of the velocity vector. Results consist of 3149 localizations assumed to originate from microbubbles coincidentally detected on both systems.

Fig. $5 \mathrm{~b}$ shows the projection of the detected localizations along the lateral direction. The two capillary tubes are clearly resolved in the elevational direction and are separated by $\sim 400$ $\mu \mathrm{m}$, which is smaller than the elevational resolution of a single transducer $(\sim 1188 \mu \mathrm{m})$ shown with red dashed vertical lines in Fig. 5c. The elevational profiles of the two tubes were found to have FWHM of $215 \mu \mathrm{m}$ and $235 \mu \mathrm{m}$, corresponding well to the nominal tube inside diameter of $200 \mu \mathrm{m}$. 


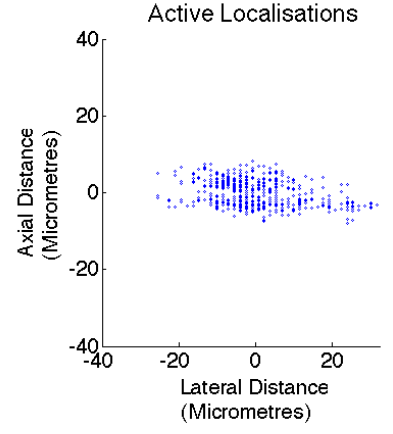

(a)

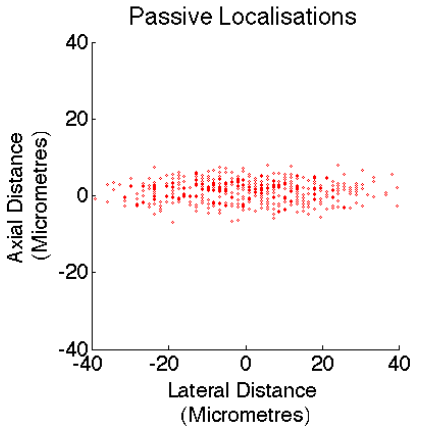

(b)

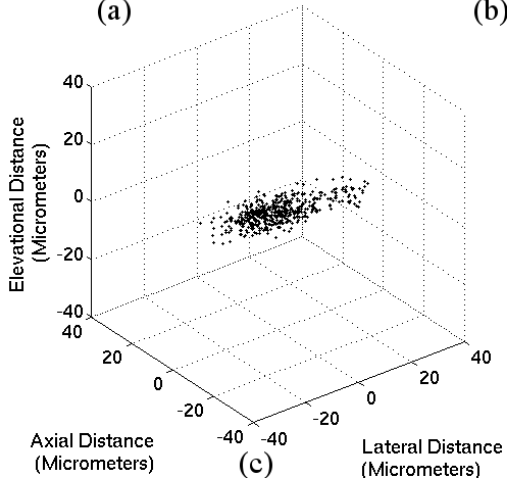

Fig. 4. 3-D localization precision. Detection by the active and passive transducers individually provides localizations as displayed in a) and b) respectively, where the axial distance in b) corresponds to the elevational distance in the active coordinate system. These figures display localizations within the transducers own co-ordinate system. When combining localizations in the global orthogonal configuration, the passive localizations enable detection within the active transducer's $(x, z)$ plane. The resulting 3-D localization precision is shown in c).

\begin{tabular}{c|c|c}
\hline \multirow{2}{*}{ Dimension } & \multicolumn{2}{|c}{ FWHM $(\boldsymbol{\mu m})$} \\
\cline { 2 - 3 } & $\begin{array}{c}\text { Conventional US } \\
\text { imaging with single } \\
\text { transducer }\end{array}$ & $\begin{array}{c}\text { Compounded } \\
\text { 3-D super-resolution }\end{array}$ \\
\hline Axial & $534 \pm 29$ & $1.9 \pm 1.6$ \\
\hline Lateral & $953 \pm 103$ & $11.0 \pm 25.0$ \\
\hline Elevational & $1188 \pm 160$ & $1.9 \pm 1.6$ \\
\hline
\end{tabular}

Table 1. Comparison of diffraction limited resolution using single LA332 transducer to $3 \mathrm{D}$ localization precision in each imaging plane of the new $3 \mathrm{D}$ orthogonal imaging configuration.

\section{DISCUSSION}

A multi-probe US strategy has been developed for superresolution imaging in 3D. This allowed detection of a single air-bubble inclusion in a paraffin gel phantom in three dimensions with average precisions of $1.9 \mu \mathrm{m}$ in the axial and elevational planes, and $11 \mu \mathrm{m}$ in the lateral plane, compared to PSF FWHM of $488 \mu \mathrm{m}, 1188 \mu \mathrm{m}$ and $953 \mu \mathrm{m}$ for the diffraction-limited resolution of a single transducer. The multi-probe compounding strategy additionally demonstrates the ability to distinguish two vessel structures in 3D far beyond the estimated diffraction limited resolution of the system, where image acquisition was performed in under 1.5 minutes. This has addressed the challenge of the existing $2 \mathrm{D}$ imaging of poor spatial resolution in the elevational plane. With additional 3D velocity mapping, investigation of both

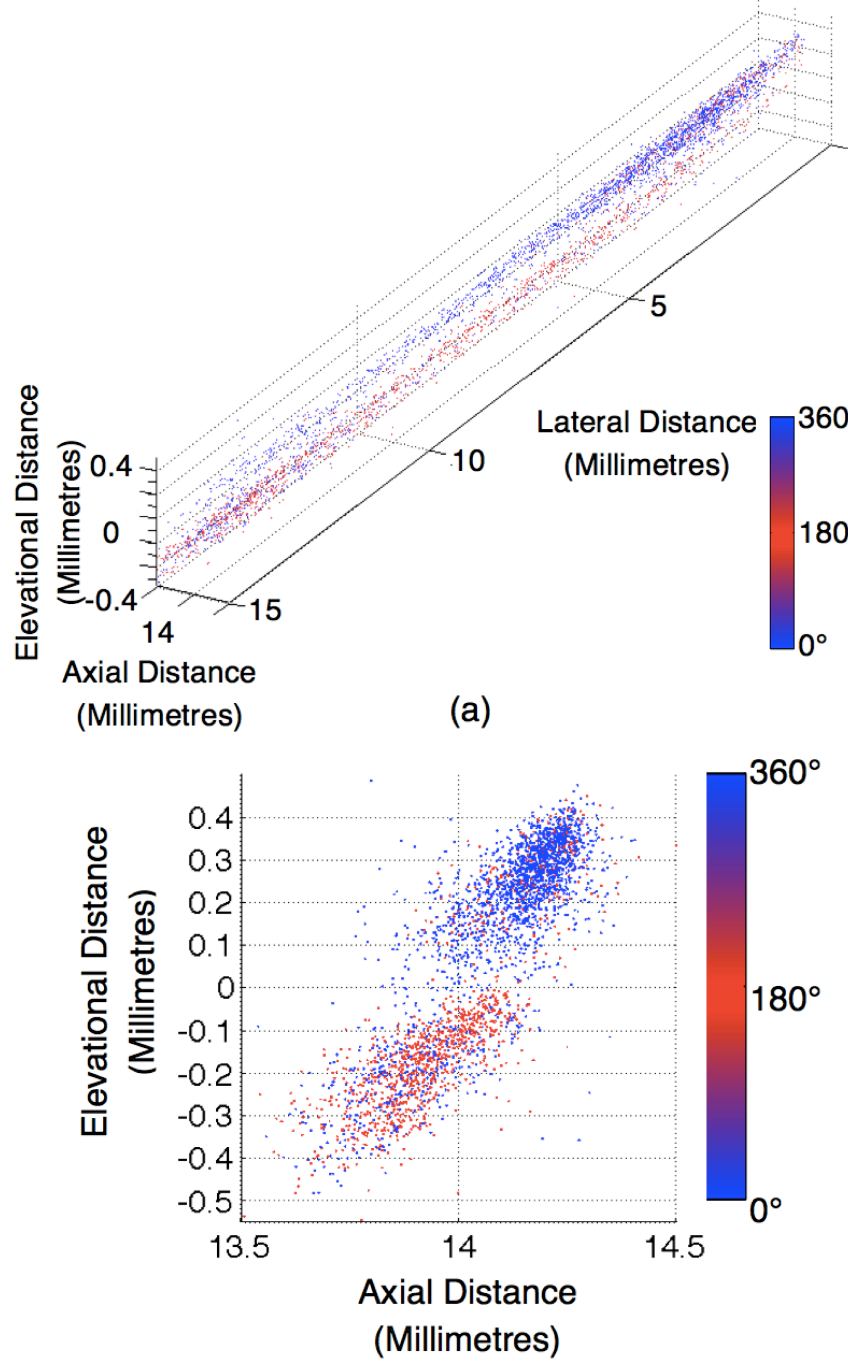

(b)

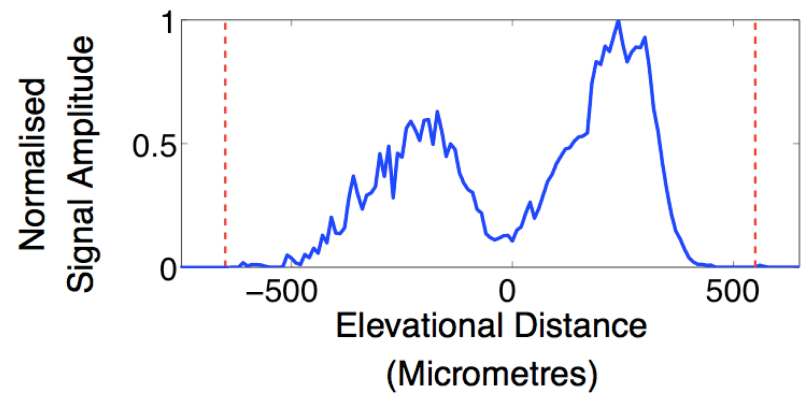

(c)

Fig. 5. Microbubble localizations after coincident detection of plane wave echoes through a flow phantom by active and passive imaging systems. a) Localizations are plotted as ellipsoids with semi-axis lengths equal to the localization precision of the system in three dimensions, $\sigma=\left(\sigma_{x}, \sigma_{x}, \sigma_{x}\right)$. The color map displays the lateral component of the velocity vector only. Fig. b) displays the projection of the microbubble localizations along the lateral plane, where color is used to display tracked direction of the axial and lateral component of velocity. Profiles of the data taken in the elevational direction, displayed in c), show the ability to resolve the two tubes within the diffraction limited elevational resolution of a single transducer $(\sim 1188 \mu \mathrm{m}-$ red dashed lines). 
structure and flow of microstructures is possible.

With such an acquisition strategy, the elevational slice thickness of each probe governs the potential 3D field of view, in that the poorer the elevational resolution, the larger the potential imaging volume; as such a poor elevational resolution becomes an asset rather than a limitation. To further increase the field of view, one or both of the systems can be scanned spatially over a larger $3 \mathrm{D}$ region of interest. For example, a mechanical probe can be used as the orthogonally placed passive receiver to detect bubble signals from a wide axial depth range. It is estimated that steering a mechanical transducer could expand the imaging volume by a factor of 2.8 at $2 \mathrm{~cm}$ depth with only a factor of three increase in acquisition time. Alternatively, imaging the target object in defocussed elevational regions of both probes should increase the overlapping imaging volume.

In comparison to our previous implementation of US-SR, the availability of the raw RF data allows both the amplitude and phase information of the US signals to be utilised, rather than only the envelope detected data. The use of plane wave RF data showed higher localization precision to that performed on beamformed reconstructed data (offline). Systems enabling raw RF data accessibility allow the more accurate detection and correction of $3 \mathrm{D}$ motion effects and may be more robust in the case of overlying, aberrating tissue [24].

In 1986, Bobroff defined the limit to which the position of a signal could be estimated for general experimental data analysis to be based on the instrument resolution, the sampling density, and the data SNR [31]. More recently, Desailly et al. 2015 extended this to the application of localisation in US [32]. Imaging parameters such as the sampling rate of the system (the temporal resolution of acquisition is predominantly limited by the system sampling frequency), the number of transducer elements, the echo duration, and the aperture size are likely to have a fundamental effect on the localization precision of RF plane wave echoes. The diffraction limited US axial resolution is proportional to the spatial pulse length (SPL) of the signal, while the lateral resolution improves with aperture length (the width of the combined number of elements used in the transducer), and increasing frequency.

The impact of the lateral position of the scatterer within the field of view on the standard deviation was investigated, and remained relatively stable within the central imaging region (Fig. 3). However, as the target neared the edge of the field of view, the lateral precision reduced considerably, where the detection of effectively only a half of the hyperbolic profile in the RF raw data was possible. SNR values were also seen to decrease for target positions near the edge of the imaging plane. Localization appeared more reliable when the targets are in the centre of the imaging volume. This super-resolution process is therefore spatially varying, and its limit will be determined by a combination of these imaging factors.
An increase in temporal resolution using plane wave imaging compared to line-scanning not only allows faster 3D image acquisition, but also should provide a higher bubble localization rate for a given microbubble concentration, and moreover, may improve velocity estimations due to more frequent sampling. Since super-resolution microbubble imaging relies upon the combined contributions of many localizations over time, for a given microbubble concentration, a greater frame rate should therefore result in a decrease in the overall acquisition time.

In order for microbubbles to provide new spatial information in each frame, the bubbles must be moving, and their position in each frame should contribute supplementary spatial information to the final rendering. As such, one can define 'additional' information to be the occurrence of a bubble localization in frame $n+1$ in which the bubble has moved by a distance of approximately half the localization precision since frame $n$. This can help to define the limit to which increasing the frame rate will no longer increase the information obtained on the spatial structure of the sample. Nevertheless, as previously mentioned, this condition does not mean increasing the frame rate will not be valuable; above this frame rate, localizations from slower moving bubbles will still contribute signal to the final rendering, and thus will enhance SNR in the final image even if they do not provide new spatial information. Indeed, the occurrence and velocity tracking of many localizations within a spatial region can importantly indicate the amount of blood flow through an area.

This study involved the use of single angle plane wave transmission. Multi-angle compounding has previously been demonstrated to provide improved SNR and lateral resolution to that of single angle transmission [18], and therefore its implementation may contribute to an improved localization precision when estimated on a stationary scatterer. However, to provide benefit, the localization precision should improve by more than the corresponding decrease in precision caused by the effect of bubble motion during the total acquisition time of the multiple compounding imaging pulses. As such, multiangle compounding in conjunction with multi-pulse contrast imaging must be considered in relation to the time taken to acquire the signal and the relative motion of the bubble during that time. The relationship between PRF, bubble velocity, and compounding strategies will be topics for future investigation.

The acceptance of only coincidental detections on the active and passive transducers is an additional noise reduction step, where noise or other false positive detections identified on one system are unlikely to occur simultaneously on the passive system also and hence may lead to a decrease in spurious localizations in the final rendering.

There, however, exist challenges associated with this imaging strategy, including the lack of suitability of the orthogonal imaging set-up for clinical implementation. Indeed, this technique can be performed when aligning the transducers at angles less than $90^{\circ}$, and this is the principle implemented 
in 2D matrix array technology used in previous publications [21]. Nonetheless, the configuration demonstrated here allows demonstration at the expected highest 3D localization precision. Implementation with smaller angles could be a topic for further investigation.

Three dimensional super-resolution techniques have been reported by two other groups, namely O'Reilly et al. 2013 [24] and Desailly et al. 2013 [21]. Both implement the use of specialized transducer technology. In the first, the use of a hemispherical transcranial therapy array where the transmit focus was steered through a volume with $2 \mathrm{~mm}$ step size allowed the impressive visualization of a spiral structure through an ex vivo skull [24]. This work demonstrated an improvement over diffraction limited US data and an average localization precision of approximately $40 \mu \mathrm{m}$ in the elevational direction is stated $(\lambda / 120)$, compared to $1.9 \mu \mathrm{m}$, $(\lambda / 263)$, in this work. Desailly et al. 2013 imaged a tilted 2D structure using a 2D matrix array transducer [21], however no analysis of the elevational resolution obtained was reported.

\section{CONCLUSION}

Three dimensional super-resolution using a synchronized, multi-probe imaging configuration allows localization of isolated bubble signals in 3D with estimated localization precision of $1.9 \mu \mathrm{m}$ in axial and elevational planes, and $11 \mu \mathrm{m}$ in the lateral plane, which is approximately two orders of magnitude higher than the original diffraction limited resolution for a single transducer $(488 \mu \mathrm{m}, 1188 \mu \mathrm{m}$ and 953 $\mu \mathrm{m}$ respectively). Plane wave imaging with a PRF of $400 \mathrm{~Hz}$ enabled a 3-D phantom consisting of two $200 \mu \mathrm{m}$ cellulose tubes with opposing flows to be imaged in 3-D with an image acquisition time of $75 \mathrm{~s}$.

\section{REFERENCES AND FOOTNOTES}

[1] K. Tuma, Ronald F. , Duran, Walter N. , Ley, Microcirculation. Academic Press, 2011.

[2] American Diabetes Association, "Peripheral Arterial Disease in People with Diabetes," Diabetes Care, vol. 26, no. 12, pp. 3333-3341, 2003.

[3] N. C. Dolan, K. Liu, M. H. Criqui, P. Greenland, J. M. Guralnik, C. Chan, J. R. Schneider, A. L. Mandapat, G. Martin, and M. M. McDermott, "Peripheral artery disease, diabetes, and reduced lower extremity functioning.," Diabetes Care, vol. 25, no. 1, pp. 113-20, Jan. 2002.

[4] R. Zatz and B. M. Brenner, "Pathogenesis of diabetic microangiopathy. The hemodynamic view," Am. J. Med., vol. 80, no. 3, pp. 443-453, Mar. 1986.

[5] M. J. Fowler, "Microvascular and Macrovascular Complications of Diabetes," Clin. Diabetes, vol. 26, no. 2, pp. 77-82, Apr. 2008.

[6] P. G. Camici and F. Crea, "Coronary Microvascular Dysfunction," N. Engl. J. Med., vol. 356, no. 8, pp. 830840, Feb. 2007.

[7] N. Weidner, J. Folkman, F. Pozza, P. Bevilacqua, E. N.
Allred, D. H. Moore, S. Meli, and G. Gasparini, "Tumor angiogenesis: a new significant and independent prognostic indicator in early-stage breast carcinoma.," $J$. Natl. Cancer Inst., vol. 84, no. 24, pp. 1875-87, Dec. 1992.

[8] D. M. McDonald and P. L. Choyke, "Imaging of angiogenesis: from microscope to clinic.," Nat. Med., vol. 9, no. 6, pp. 713-25, Jun. 2003.

[9] N. Weidner, "Intratumor microvessel density as a prognostic factor in cancer.," Am. J. Pathol., vol. 147, no. 1, pp. 9-19, Jul. 1995.

[10] N. Weidner, J. P. Semple, W. R. Welch, and J. Folkman, "Tumor angiogenesis and metastasis--correlation in invasive breast carcinoma.," N. Engl. J. Med., vol. 324, no. 1, pp. 1-8, Jan. 1991.

[11] D. E. Goertz, D. A. Christopher, J. L. Yu, R. S. Kerbel, P. N. Burns, and F. S. Foster, "High-frequency color flow imaging of the microcirculation.," Ultrasound Med. Biol., vol. 26, no. 1, pp. 63-71, Jan. 2000.

[12] H. Becher and P. N. Burns, Handbook of contrast echocardiography: left ventricular function and myocardial perfusion. Springer, 2000.

[13] D. Cosgrove and N. Lassau, "Imaging of perfusion using ultrasound.," Eur. J. Nucl. Med. Mol. Imaging, vol. 37 Suppl 1, pp. S65-85, Aug. 2010.

[14] T. S. Desser and R. B. Jeffrey, "Tissue harmonic imaging techniques: physical principles and clinical applications.," Semin. Ultrasound. CT. MR, vol. 22, no. 1, pp. 1-10, Feb. 2001.

[15] O. M. Viessmann, R. J. Eckersley, K. ChristensenJeffries, M. X. Tang, and C. Dunsby, "Acoustic superresolution with ultrasound and microbubbles.," Phys. Med. Biol., vol. 58, no. 18, pp. 6447-58, Sep. 2013.

[16] K. Christensen-Jeffries, R. J. Browning, M.-X. Tang, C. Dunsby, and R. J. Eckersley, "In Vivo Acoustic SuperResolution and Super-Resolved Velocity Mapping Using Microbubbles," IEEE Trans. Med. Imaging, 2015.

[17] M. Tanter, J. Bercoff, L. Sandrin, and M. Fink, "Ultrafast compound imaging for 2-D motion vector estimation: application to transient elastography," IEEE Trans. Ultrason. Ferroelectr. Freq. Control, vol. 49, no. 10, pp. 1363-1374, Oct. 2002.

[18] G. Montaldo, M. Tanter, J. Bercoff, N. Benech, and M. Fink, "Coherent plane-wave compounding for very high frame rate ultrasonography and transient elastography," IEEE Trans. Ultrason. Ferroelectr. Freq. Control, 2009.

[19] M. Tanter and M. Fink, "Ultrafast imaging in biomedical ultrasound," IEEE Trans. Ultrason. Ferroelectr. Freq. Control, vol. 61, no. 1, pp. 102-119, Jan. 2014.

[20] O. Couture, S. Bannouf, G. Montaldo, J.-F. Aubry, M. Fink, and M. Tanter, "Ultrafast Imaging of Ultrasound Contrast Agents," Ultrasound Med. Biol., vol. 35, no. 11, pp. 1908-1916, Nov. 2009.

[21] Y. Desailly, O. Couture, M. Fink, and M. Tanter, "Sonoactivated ultrasound localization microscopy," Appl. Phys. Lett., vol. 103, no. 17, 2013.

[22] C. Errico, J. Pierre, S. Pezet, Y. Desailly, Z. Lenkei, O. 
Couture, and M. Tanter, "Ultrafast ultrasound localization microscopy for deep super-resolution vascular imaging.," Nature, vol. 527, no. 7579, pp. 499$502,2015$.

[23] D. Ackermann, G. Schmitz, and S. Member, "Detection and Tracking of Multiple Microbubbles in Ultrasound BMode Images," vol. 63, no. 1, pp. 72-82, 2016.

[24] M. a O'Reilly and K. Hynynen, "A super-resolution ultrasound method for brain vascular mapping.," Med. Phys., vol. 40, no. 11, p. 110701, Nov. 2013.

[25] S. Hilgenfeldt, D. Lohse, and M. Zomack, "Response of bubbles to diagnostic ultrasound: a unifying theoretical approach," Eur. Phys. J. B, vol. 4, no. 2, pp. 247-255, Aug. 1998.

[26] P. J. Phillips, “Contrast pulse sequences (CPS): imaging nonlinear microbubbles," in 2001 IEEE Ultrasonics Symposium. Proceedings. An International Symposium (Cat. No.01CH37263), 2001, vol. 2, pp. 1739-1745.

[27] M. Gyöngy and C.-C. Coussios, "Passive cavitation mapping for localization and tracking of bubble dynamics," J. Acoust. Soc. Am., vol. 128, no. 4, p. EL175-EL180, Oct. 2010.

[28] P. Tortoli, S. Member, L. Bassi, E. Boni, A. Dallai, F. Guidi, and S. Ricci, "ULA-OP: An Advanced Open Platform for Ultrasound Research," vol. 56, no. 10, pp. 2207-2216, 2009.

[29] S. L. Vieira, T. Z. Pavan, J. E. Junior, and A. a O. Carneiro, "Paraffin-Gel Tissue-Mimicking Material for Ultrasound-Guided Needle Biopsy Phantom," Ultrasound Med. Biol., vol. 39, no. 12, pp. 2477-2484, 2013.

[30] C. Sun, S. D. Pye, J. E. Browne, A. Janeczko, B. Ellis, M. B. Butler, V. Sboros, A. J. W. Thomson, M. P. Brewin, C. H. Earnshaw, and C. M. Moran, "The speed of sound and attenuation of an IEC agar-based tissue-mimicking material for high frequency ultrasound applications.," Ultrasound Med. Biol., vol. 38, no. 7, pp. 1262-70, Jul. 2012.

[31] N. Bobroff, "Position measurement with a resolution and noise-limited instrument," Rev. Sci. Instrum., vol. 57, no. 6, p. 1152, Jun. 1986.

[32] Y. Desailly, J. Pierre, O. Couture, and M. Tanter, "Resolution limits of ultrafast ultrasound localization microscopy," Phys. Med. Biol., vol. 60, no. 22, pp. 872340, 2015.

The data supporting this research are openly available from the King's College London research data archive at doi:10.18742/RDM01-210. 